\title{
Hepatectomía en dos tiempos con resección colorrectal simultánea abierta en el tratamiento multimodal de las metástasis hepáticas de origen colorrectal Two stage hepatectomy with simultaneous open colon resection for multimodal treatment of colorec- tal liver metastases
}

\author{
Gustavo A. Nari, José Layún, Lino Molina, Luis Barrionuevo, Elías Ortega, Alesio López
}

Servicio de Cirugía de Hospital Tránsito

Cáceres de Allende.

Córdoba. Argentina

Los autores declaran no tener conflictos de interes

Correspondencia

Gustavo A. Nari e-mail: gusnari@hotmail.com

\section{RESUMEN}

Antecedentes: los mejores resultados ante la presencia de metástasis (MTS) hepáticas de origen colorrectal los ofrece la resección hepática. Aproximadamente el $50 \%$ de los pacientes afectados de cáncer de colon y recto presentará metástasis hepáticas en el transcurso de la enfermedad. Un grupo de pacientes con metástasis bilobares consideradas irresecables o marginales hace un tiempo pueden, en la actualidad, ingresar en protocolos de resecabilidad con estrategias que involucran resecciones hepáticas escalonadas en dos tiempos con quimioterapia neoadyuvante o sin ella.

Objetivo: mostrar los resultados obtenidos en 3 pacientes con metástasis bilobares de origen colorrectal sometidos a este protocolo con el distintivo de la resección simultánea colónica en el primer paso. Material y métodos: se presentan tres pacientes tratados con una estrategia quirúrgica en dos tiempos por metástasis hepáticas simultáneas de origen colorrectal. Se analizan los datos demográficos, del tumor y los correspondientes a las resecciones.

Resultados: los tres pacientes pudieron completar el tratamiento. No hubo mortalidad operatoria. No hubo morbilidad en el primer paso de la resección y sí hubo una complicación en el segundo paso. La supervivencia tiene una mediana de 16,3 meses y un período libre de enfermedad de 10,6 meses. Hubo recurrencia en un solo paciente.

Conclusión: la estrategia en dos tiempos con resección simultánea en el primer paso es segura. Ofrece una alternativa de curación a estos pacientes o en su defecto una franca prolongación de su sobrevida cuando se la asocia a quimioterapia u otras estrategias.

Palabras clave: metástasis colorrectal, hepatectomía en dos tiempos.

ABSTRACT

Background: the best results for hepatic metastasis of colorectal cancer are offered by hepatic resection. Approximately $50 \%$ of patients with colon and rectum cancer will develop liver metastases in the course of the disease. Bi-lobular metastases, previously considered to be unresectable may be treated by two stage stage liver resection with or without neoadjuvant chemotherapy.

Objective: to describe the results upon treatment of bi-lobular colorectal metastases with a two stage strategy in which a simultaneous colon resection was done at the first stage.

Material and methods: three patients were treated with a two stage strategy for simultaneous hepatic metastases of colorectal cancer. Demographic, tumor, and resection data were analyzed.

Results: all three patients were able to complete the treatment. No operative mortality occurred. There was no morbidity during the first step of the resection while a complication was seen during the second step. Survival and disease free time were 16.3 months and 10.6 months, respectively. To date, recurrence was seen in only one patient.

Conclusion: the two stage strategy with simultaneous colon resection in the first step is safe and may offer a chance for cure or a prolongation of survival when associated with chemotherapy or other treatment strategies.

Keywords: colorectal metastases, liver resection. 


\section{Introducción}

La resección hepática sigue siendo la mejor alternativa en el tratamiento de las metástasis de origen colorrectal. La seguridad de estas resecciones asociada a una mejoría en los resultados de los agentes quimioterápicos ha permitido que con estrategias de tipo multimodal un mayor número de pacientes con enfermedad considerada irresecable o "marginal" puedan acceder al tratamiento quirúrgico. En el año 2000, el equipo del Hospital Paul Brousse ${ }^{1}$ propuso las hepatectomías en dos tiempos o "two stage" para resolver tumores hepáticos que por su número y localización eran considerados irresecables. En ese trabajo, los autores proponen como objetivo de la primera hepatectomía hacer posible una segunda con carácter curativo; para esto plantean dos alternativas, la resección del mayor número de metástasis o la limpieza del lóbulo menos afectado seguido de quimioterapia y selección del paciente que sería sometido a una segunda hepatectomía entre aquellos que respondían a la quimioterapia o tenían enfermedad estable. El tiempo de esta segunda resección estaba supeditado al tiempo que requería la regeneración hepática. En 2004, el equipo de Daniel $J$ aeck $^{2}$ propone la limpieza de un lóbulo con la manipulación del flujo portal (embolización) para favorecer la hipertrofia compensadora del futuro remanente hepático y la consiguiente disminución de los riesgos de insuficiencia hepática posoperatoria en el segundo tiempo quirúrgico.

Posteriormente algunos autores sugirieron que la ligadura de la vena porta del hemihígado para resecar en el segundo paso lograba una hipertrofia de características similares a la lograda con la embolización ${ }^{3,4}$.

Con el incremento de la experiencia, las hepatectomías en dos tiempos se transformaron en parte del arsenal terapéutico para el tratamiento de las metástasis hepáticas de origen colorrectal.

El objetivo de este trabajo es presentar los resultados obtenidos en 3 pacientes sometidos a estrategias de resecciones en dos tiempos.

\section{Material y métodos}

Se presentan 3 pacientes sometidos a estrategia de resección hepática en dos tiempos. Los pacientes firmaron el consentimiento informado una vez que comprendieron los procedimientos a los que serían sometidos. La decisión de ingresarlos a un protocolo de resección en 2 tiempos fue tomada en un comité de tumores.

Técnica operatoria: el primer tiempo quirúrgico consistió en el abordaje de la glándula hepática con la resección de las lesiones del lóbulo izquierdo y ecografía intraoperatoria para garantizar, en lo posible, la ausen- cia de lesiones residuales en esa región. Se efectuó ligadura de la vena porta del hemihígado derecho. En el primer paso se trató de realizar la menor disección posible a nivel hiliar y hepático apuntando a disminuir las dificultades de una posible segunda resección. Posteriormente se efectuó la resección de la patología colorrectal.

El segundo tiempo consistió en la resección del hemihígado derecho con la enfermedad residual. Las resecciones hepáticas se efectuaron con la técnica denominada "kellyclasia". En las resecciones limitadas realizadas en el primer paso se utilizó la técnica del "tirabuzón". La resección derecha del segundo tiempo se efectuó ligando la arteria hepática derecha y el conducto hepático derecho inicialmente, mientras que la ligadura de la vena suprahepática derecha se efectuó de forma transparenquimatosa.

Los datos evaluados fueron: sexo, edad, localización del tumor primario y datos inherentes a su estadio y resección, volumen del futuro remanente hepático (FRH) preoperatorio y posterior primer paso con tomografía computarizada multicorte con inyección de contraste intravenoso (I.V.), uso de quimioterapia interresecciones, complicaciones y su gravedad según la clasificación de Dindo-Clavien ${ }^{5}$, estancia hospitalaria en días, uso de alcoholización o radiofrecuencia, nuevas resecciones, sobrevida y período libre de enfermedad en meses. En caso de fístula biliar se utilizó la clasificación del International Study Group of Liver Surgery (ISGLS) 6 y en caso de insuficiencia hepática posoperatoria las clasificación del 50/507.

\section{Resultados}

En los últimos 8 años fueron intervenidos por un solo cirujano 83 pacientes por metástasis de origen colorrectal con un notorio incremento de casos en los últimos 4 años, lo que motivó que en los últimos 3 años se decidiera avanzar en procedimientos de mayor complejidad como las resecciones en dos tiempos.

En los últimos 3 años fueron sometidos a una hepatectomía en 2 tiempos 3 pacientes, los 3 fueron de sexo masculino con una mediana de edad de 58 años. En los 3 pacientes se realizó quimioterapia neoadyuvante con 3 ciclos de FOLFOX con respuesta parcial según el RECIST 1.1. Todos los pacientes hicieron adyuvancia.

En dos pacientes, la resección colónica fue seguida de anastomosis primaria y los 3 pacientes durante la primera resección fueron incluidos en un programa ERAS. Los datos demográficos así como algunos de los datos referentes al tumor primario se muestran en la tabla 1. Los márgenes de la resección colónica estuvieron libres al examen patológico; en los 3 pacientes fue un adenocarcinoma moderadamente diferenciado y en los 3 hubo invasión vascular, infiltración perineural y permeación linfática. El promedio de días entre la primera y la segunda cirugía fue de 59; en un solo paciente 
se efectuó un ciclo de quimioterapia en el intermedio de las cirugías. No hubo morbimortalidad en el primer tiempo quirúrgico, mientras que, en el segundo, uno de los pacientes presentó una evisceración aguda que requirió una nueva intervención al $4^{\circ}$ día de posoperatorio (complicación tipo IIIb de Dindo y Clavien), en la que se le reparó la eventración con técnica mixta con malla y procedimiento de Clotteau-Premont (morbilidad de 33,3\%). En los 3 pacientes se utilizó la maniobra de Pringle en el segundo tiempo; en uno de ellos se utilizó una hanging maneuver a la hora de efectuar la hemihepatectomía derecha. Dos pacientes recibieron transfusión de una y dos unidades de sangre, respectivamente. Los márgenes de las resecciones hepáticas del primero y segundo paso estuvieron libres de tumor en todos los casos. No hubo mortalidad en el segundo tiempo. La mediana de supervivencia es de 16, 3 meses con un período libre de enfermedad de 10,6 meses. Un paciente presentó una recurrencia de 3 lesiones a los 11 meses de la $2^{\text {a }}$ cirugía y fue nuevamente resecado de dos de ellas y la $3^{a}$ lesión fue alcoholizada por no contar en ese momento con la posibilidad de realizar radiofrecuencia y por la dificultad técnica para resecarla; en este mismo paciente hubo recurrencia pulmonar y hepática 7 meses después de la rehepatectomía. En las tablas 2 y 3 se presentan algunos datos inherentes a las resecciones hepáticas.

\section{Discusión}

Las resecciones hepáticas continúan siendo el procedimiento más eficaz en el tratamiento de las metástasis de origen colorrectal. El advenimiento de estrategias multimodales ha logrado prolongar la sobrevida de los pacientes e incrementar el número que accede a esta posibilidad. Entre las estrategias que permiten incrementar las posibilidades se encuentran aquellas que involucran la resección en dos tiempos ${ }^{8}$.

Adhiriendo a esta tendencia desde hace 3 años incorporamos la aplicación de las hepatectomías en dos tiempos para nuestros pacientes con metástasis hepáticas de origen colorrectal. Los tres pacientes recibieron quimioterapia de conversión; en los 3 se realizó volumetría hepática con una mediana para el FRH de $30 \%$, pese a lo cual decidimos incluirlos en el proceso porque consideramos que el $30 \%$ con lesiones y con quimioterapia preoperatoria corren el riesgo de desarrollar un episodio de insuficiencia hepática en el posoperatorio de la segunda resección. Durante la primera resección no tuvimos morbimortalidad y los 3 pacientes fueron incorporados en un programa ERAS ${ }^{9}$. Algunos autores refieren una morbilidad de entre el 11 y el $17 \%$ para la primera resección con mortalidad prácticamente nula ${ }^{10}$, mientras que Torzilli y col..$^{11}$ refieren una mortalidad del $0,8 \%$ en la primera resección

IABLA 1

Datos demográficos y referentes al tumor primario

\begin{tabular}{lcccccc}
\hline Sexo & Edad & T. primario & $\begin{array}{c}\text { Quimioterapia } \\
\text { neoadyuvante }\end{array}$ & $\begin{array}{c}\text { Anastomosis } \\
\text { primaria }\end{array}$ & $\begin{array}{c}\text { pTNM - } \\
\text { AJCC }\end{array}$ & $\begin{array}{c}\text { Relac-Ganglios } \\
\text { Positivos/Totales }\end{array}$ \\
Masculino & 54 & Recto & Sí. FOLFOX & Sí. Manual & T3N1M1a & $3 / 160,18$ \\
Masculino & 74 & Sigmoides & Sí. FOLFOX & Sí. Manual & T3NOM1a & $0 / 110,00$ \\
Masculino & 47 & Sigmoides & Sí. FOLFOX & No & T3N2aM1a & $4 / 140,28$ \\
3 & 58,3 & $66,6 \%$ & $100 \%$ & $66,6 \%$ & & \\
\hline
\end{tabular}

I TABLA 2

Resultados de la primera y segunda resección hepática

\begin{tabular}{lcccccc}
\hline $\begin{array}{l}\text { FRH pre- } \\
\text { primer paso }\end{array}$ & $\begin{array}{c}\text { No MTS } 1^{\mathrm{a}} \\
\text { resección }\end{array}$ & $\begin{array}{c}\text { Estancia hosp } \\
1^{\mathrm{a}} \text { resección }\end{array}$ & $\begin{array}{c}\text { Tiempo entre } \\
1^{\mathrm{a}} \text { y } 2^{\mathrm{a}} \text { res. }\end{array}$ & $\begin{array}{c}\text { FRH pos lig. } \\
\text { portal }\end{array}$ & $\begin{array}{c}\text { Qt. inter } \\
\text { resección }\end{array}$ & $\begin{array}{c}\text { Estancia hosp } \\
2^{\mathrm{a}} \text { resección }\end{array}$ \\
\hline $28 \%$ & 4 & 3 días ERAS & 56 días & $39 \%$ & Sí & 6 \\
$30 \%$ & 4 & 4 días ERAS & 62 días & $42 \%$ & No & 13 \\
$32 \%$ & 3 & 3 días ERAS & 60 días & $42 \%$ & No & 6 \\
$30 \%$ & 3,6 & 3,3 & 59,3 días & $41 \%$ & $66,6 \%$ & 8,3 días \\
\hline
\end{tabular}

TABLA 3

Resultados de la primera y segunda resección hepática

\begin{tabular}{lccccc}
\hline Complicación & Reintervención & $\begin{array}{c}3^{\text {a }} \text { re- } \\
\text { hepatectomía }\end{array}$ & Alcoholización & Sobrevida & $\begin{array}{c}\text { Tiempo Libre } \\
\text { de enfermedad }\end{array}$ \\
\hline No & No & Sí ERAS & Sí & 28 meses & 11 meses \\
Sí Evisceración & Sí & No & No & 14 meses & 14 meses \\
No & No & No & No & 7 meses & 7 meses \\
$66,6 \%$ & $33,3 \%$ & $33,3 \%$ & $33,3 \%$ & 16,3 meses & 10,6 meses \\
\hline
\end{tabular}


pero no informan si hubo resecciones simultáneas que pudieran incrementar la morbimortalidad. El promedio de lesiones resecadas en el primer paso en nuestros pacientes fue de 3,6 lesiones; creemos que, en la medida en que uno pueda lograr la resección completa de las lesiones, su número no debe ser una limitante. Por otra parte, el uso de la ecografía intraoperatoria es de gran ayuda, aunque a pesar de su uso se considera que en alrededor de un $5 \%$ de los pacientes existen "missing" metástasis que favorecerán una recaída ${ }^{12}$. En otro sentido, algunos autores hallaron que los pacientes con tumores colorrectales tipo Dukes C y de sexo masculino tienen mayor incidencia de recaída temprana a nivel hepático, mientras que aquellos que tienen metástasis con un tamaño mayor de $3,6 \mathrm{~cm}$ y más de 2 lesiones desarrollan un mayor porcentaje de recurrencia sistémica ${ }^{13}$.

En el intervalo entre la primera y la segunda resección utilizamos quimioterapia en un solo paciente; su uso -a nuestro entender- presenta ventajas y desventajas que hacen que aún sea discutida. Como ventaja podemos entender que el uso de quimioterapia puede evitar la progresión de la enfermedad en este período de tiempo y evitar que un paciente quede sin chances de completar el tratamiento; como desventaja puede hacer invisibles lesiones pequeñas y favorecer la recurrencia posoperatoria en el FRH. El promedio de días entre la primera y la segunda resección fue de 59 días, lo que no difiere de lo informado en la bibliografía $^{8,13}$, mientras que el incremento del FRH posligadura de la vena porta tuvo una media de $41 \%$ principalmente a expensas del segmento IV.

La segunda resección pudo realizarse en los tres pacientes. La mayoría de los autores refieren que esta puede llevarse a cabo en entre el 76 y el $87 \%^{14,15}$ de los casos; probablemente con el aumento de nuestra experiencia tengamos pacientes con progresión intratratamiento que no puedan acceder a la segunda resección y nuestros porcentajes se aproximen a los de series con más volumen. Los tres pacientes fueron considerados R0. Diferentes autores informan cifras entre 58 y $79 \%$ donde logran este tipo de resección ${ }^{2,15}$; es probable que el porcentaje RO obtenido tenga una relación importante con la selección de pacientes.

Uno de nuestros pacientes presentó en el posoperatorio una evisceración aguda que requirió una nueva intervención, que representó la única complicación. No tuvimos mortalidad a los 90 días; aunque va- rios autores refieren una mortalidad de 2,5\% durante la segunda hepatectomía, esta no difiere de la de las hepatectomías mayores. El tiempo de estancia hospitalaria tuvo una media de 8,3 días, que no difiere de lo publicado ${ }^{8}$.

Cuando analizamos la sobrevida, nuestros pacientes tienen a la fecha una sobrevida de 16,3 meses en promedio y un período libre de enfermedad de 10,6 meses. Algunos autores informan una sobrevida a 3 años del 50 al $84 \%^{2,14,15}$, mientras que otros refieren una supervivencia a 5 años de entre el 32 y el $64 \%{ }^{11}$. Al igual que lo que pensamos de las resecciones R0, es probable que la supervivencia dependa de la selección de los pacientes.

Es importante destacar que aquellos pacientes que logran completar los dos pasos de resección tienen una sobrevida mayor que aquellos que no completaron el tratamiento ${ }^{11}$; por otra parte aquellos pacientes que no han sido incluidos en ningún protocolo resectivo tienen una sobrevida escasa, lo que estimula a incorporar a través de quimioterapia de conversión a la mayor cantidad de pacientes. En otro sentido y atendiendo a los buenos resultados obtenidos con las re-resecciones hepáticas, esta estrategia debe contarse como una posibilidad. Nosotros la hemos utilizado en un enfermo que, aun con recurrencia de la enfermedad, tiene una sobrevida de 28 meses en muy buen estado general. Se están implementando nuevas estrategias que aceleran el tiempo entre la primera y la segunda resección (ALPPS), donde en el momento de la primera cirugía y ligadura portal se realiza la partición hepática ${ }^{16}$. El fundamento es que, después de la partición, se estimula de una manera más rápida la hipertrofia del FRH permitiendo acortar el tiempo intercirugías. Inicialmente, este procedimiento tuvo alta morbimortalidad, que ha ido disminuyendo con el aumento de la experiencia. Aún quedarían por resolverse el aumento de las recurrencias tempranas y valorar adecuadamente la madurez de los hepatocitos regenerados ${ }^{17}$.

En conclusión, a pesar de ser una experiencia pequeña, nuestros resultados coinciden con la literatura y nos impresiona que las resecciones en dos tiempos son factibles, prolongan la supervivencia de los pacientes y la morbimortalidad no difiere de la que se registra en las que se hacen en un solo tiempo. Todos estos factores permiten un aumento del número de pacientes que pueden beneficiarse y que antes eran considerados irresecables.
1. Adam R, Laurent A, Azoulay D, Castaing D, Bismuth $\mathrm{H}$. Two Stage Hepatectomy: A planned Strategy to treat irresectable liver tumors. Ann Surg. 2000; 232:777-85.

2. Jaeck D, Oussoutzoglou E, Rosso E, Greger M, Weber J, Bachellier P. A two stage hepatectomy procedure combined with portal vein embolization to achieve curative resection for initially unresectable multiple and bilateral colorectal liver metastases. Ann Surg. 2004; 240:1057-63.

3. Kianmanesh R, Farges O, Abdalla E, Sauvanet A, Rusznieski P, et al.
Right portal vein ligation: a new planned two stepall-surgical approach for complete resection of primary gastrointestinal tumors with multiple bilateral liver metastases. J Am Coll Surg. 2003; 197:164-70.

4. Aussilhou B, Lesurte $M$, Sauvanet A, et al. Right portal vein ligation is a efficient as portal vein embolization hipertruphy of the left liver remant. J Gastrointest Surg. 2008; 12:297-303.

5. Dindo D, Clavien P. Classification of surgical complications. A new proposal with evaluation in a cohort od 6336 
patients and results od a survey. Ann Surg. 2004; 240:205-13. 6. Koch M, Garden J, Padbury R, Rahbari M, Adm R, Capusotti L, et al. Bile leakage after hepatobiliary and pancreatic surgery: a definition and grady of severity by the International Study Group of Liver Surgery. Surgery. 2011; 149:680-8.

7. Balzan S, Belghitti J, Farges O, Ogata S, Sauvanet A, Delafosse D et al. The $50 / 50$ criteria on postoperative day 5. Ann Surg. 2005; 242:824-29.

8. Andriani O, González Campaña A, Fauda M, Rowe C. Cirugía de remodelación hepática para el tratamiento de las metástasis inicialmente resecables. Rev Argent Cirug. 2010; 98:142-51.

9. Nari G, Molina L, Gil F, Viotto L, Layún J, Mariot D, et al. Enhanced recovery after surgery (ERAS) en resecciones hepáticas abiertas por metástasis de origen colorrectal. Experiencia inicial. Rev Argent Cirug. 2016; 108:9-13.

10. Clark M, Smith R. Liver directed therapies in metastatic colorectal cancer. J Gastrointest Oncol. 2014; 5:374-87.

11. Torzill G, Adam R, Vigano L, Imai K, Goransky J, et al. Surgery of colorectal liver metastases: pushing the limits. Liver Cancer. 2017; 6:80-9.

12. Castro Ribeiro H, Martins Torres O, Carmona Marques M, Herman $P$, Nocchi Kalil A, Martins Fernandes E, et al. I Brazilian Consensus on multimodal treatment of colorectal Liver metastases. Module 2: approach to resectable metastases. Arq Bras Cir Dig. 2016 29:9-13.

13. Bhogal R, Hodson J, Bramhall S, Isaac J, Marudanayagam R, Mirza $\mathrm{D}$, et al. Predictor of early recurrence after resection of colorectal liver metastases. World J SurgOncol. 2015; 13:135-41.

14. Brouquet A, Abdalla EK, Kopetz $S$, et al. High survival rate after two-stage resection of advanced colorectal liver metastases: Response-based selection and complete resection define outcome. J Clin Oncol. 2011; 29:1083-90.

15. Tsim N, Healey AJ, Frampton AE, et al. Two-stage resection for bilobar colorectal liver metastases: RO resection is the key. Ann Surg Oncol. 2011; 18:1939-46.

16. Schadde E, Ardiles V, Robles Campo R, Malago M, Machado M, Hernández-Alejandro R, et al. Early survival and safety of ALPPS: first report of International ALPPS registry. Ann Surg. 2014; 260:828-36.

17. Matsuo K, Murakami T, Kawaguchi D, Hiroshima Y, Koda K, Yamazaki $\mathrm{K}$, et al. Histologic features after surgery associating liver partition and portal vein ligation for staged hepatectomy Vs those after hepatectomy with portal vein embolization. Surgery. 2016: 159:1289-98. 